\title{
Nativos digitales y brecha digital: una visión comparativa en el uso de las TIC
}

Digital natives and the digital divide: a comparative overview in ICT use

\section{Juan-Francisco Álvarez-Sigüenza}

Universidad de Cádiz

juan.alvarezsi@alum.uca.es

Forma de citar este artículo:

Álvarez-Sigüenza, J. F. (2018). “Nativos digitales y brecha digital: una visión comparativa en el uso de las TIC", RAEIC, Revista de la Asociación Española de Investigación de la Comunicación, vol 6, núm. 11, 203-223.

DOI: https://doi.org/10.24137/raeic.6.11.12

\section{Resumen:}

Los avances tecnológicos y el acceso a ellas de los denominados nativos digitales han creado una nueva forma de vida creadas entorno a lo digital, utilizan los medios y sus contactos principalmente a través de las redes sociales para interaccionar con altos índices de actividad; fuera de estos conceptos se encuentra otra generación incluida en lo que puede definirse como brecha digital. El análisis de los datos vertidos por el Instituto Español de Estadística ayudará mejor a conocer y comprender estos conceptos.

Palabras clave: Nativo, brecha, digital, tecnologías. 


\section{Abstract:}

The technological advances and access to them of the so-called digital natives have created a new way of life created around the digital, they use the media and their contacts mainly through social networks to interact with high indices of activity; out of these concepts finds another generation included in what can be defined as digital divide. The analysis of the data discharged by the Spanish Institute of Statistics will help better to know and understand these concepts.

Keywords: Native, gap, digital, technologies.

\section{INTRODUCCIÓN}

El presente estudio es un análisis sobre las nuevas formas de comunicación emergentes, el efecto que ha tenido en la ciudadanía el mundo digital con la creación de diversos grupos sociales tras la adopción de las TIC en la sociedad actual.

El marco teórico está compuesto por las definiciones y teorías relacionadas con los nativos digitales y la brecha digital de edad, género y de condición social.

La problemática generada por la dificultad de solucionar las contingencias generadas por la difícil adaptación de las personas de más edad a las TIC, la diferencia entre hombres y mujeres y la dificultad de acceso de las personas o familias con menor ingreso, unido a que en cierta medida los denominados nativos digitales en un efecto de empoderamiento de las redes, hacen que se haya generado un cúmulo de circunstancias que hacen difícil la convivencia y la sostenibilidad entre las generaciones y géneros.

Los antecedentes encontrados para el presente estudio son muy diversos y han sido tratados por diferentes autores, cada uno dando un enfoque distinto del impacto de las TIC en la educación, acceso a los medios o desigualdad de oportunidades; autores como Mónica Arenas Ramiro en su estudio sobre la Brecha digital de género centrados en la mujer y las nuevas tecnologías estudia la desigualdad de oportunidades que se han generado con las TIC en el sexo femenino, otros autores como Begoña Peral Peral, Jorge 
Arenas Gaitán y Ángel Francisco Villarejo-Ramos en su artículo De la brecha digital a la brecha psico-digital: Mayores y redes sociales, estudia el impacto negativo y positivo de las nuevas tecnologías en la población de más edad centrado en las variables psicológicas surgidas en su muestra, otros estudios como el de la autora Sofía Olarte Encabo titulada Brecha digital, pobreza y exclusión social, analiza en palabras de ella misma "el impacto de la sociedad tecnológica desde la perspectiva del principio de igualdad y no discriminación. Para ello se estudian los conceptos y elementos definitorios de la revolución tecnológica y la brecha digital" (Olarte, 2017, p. 285), otros casos como el de los nativos digitales ha sido estudiado por autores como Manuel Gertrudix-Barrios en su obra Nativos digitales resume el origen del término y cómo se comporta esta generación totalmente inserta y familiarizada con las TIC.

Las razones que han llevado a los autores a realizar un trabajo de análisis y a pronunciarse en estos temas, ha sido la consideración de que es un tema que merece un tratamiento adecuado, de especial interés y actualidad y una búsqueda de soluciones a las diferentes problemáticas que se han generado en la sociedad en general y en la sociedad de la información.

Es importante estudiar estos temas, ya que es de primera actualidad y la sociedad actual con adopción de las TIC en todos los sectores ha provocado que ninguna persona independientemente de edad, género o condición social se vea excluida del impacto de la inserción de las nuevas tecnologías en sus actividades sociales, laborales y personales.

El objetivo que se ha propuesto a lo largo del presente artículo es conocer y comprender los conceptos de nativo digital y brecha digital, desde una perspectiva actualizada y tomando como muestra en el caso de los nativos digitales la franja de edad comprendida entre las personas con una edad comprendida entre los 16 y 24 años y en el caso de los ciudadanos insertos en la brecha digital las personas con una edad comprendida entre los 54 y 65 años, ya que se ha considerado que es una muestra más representativa dado que son personas con capacidad de obrar y en edad laboral, las diferencias entre hombres y mujeres y el uso de las TIC de las familias con ingresos mensuales netos del 
hogar al que pertenecen de menos de 900 euros y las familias con ingresos mensuales netos del hogar al que pertenecen de más de 2.500 euros.

La metodología empleada para el análisis será la cuantitativa, los resultados del análisis de las muestras, en este caso son una selección de encuestas realizadas por el Instituto Nacional de Estadística de España, sobre equipamiento y uso de Tecnologías de la Comunicación y la Información en los hogares durante el 2017, todas ellas contienen información sobre hábitos de uso, franjas salariales, sobre equipamientos tecnológicos digitales en los hogares y usos de Internet, franjas de edad que usan equipamiento digital, franjas de género entre hombres y mujeres.

A priori se esperan resultados que darán clara muestra de cómo ha impactado y se ha comportado la ciudadanía española ante las Tecnologías de la Información y de la Comunicación que ayudarán comprender y conocer mejor los conceptos planteados.

Las conclusiones se centrarán en los principales puntos diferenciadores que ayudarán a comprender mejor el objetivo propuesto.

\section{MARCO TEÓRICO}

\subsection{NATIVOS DIGITALES}

Los nativos digitales, es un término:

\section{[...] acuñado por Mark Prensky [...] forma parte ya del imaginario colectivo de} nuestra sociedad, al designar a aquellos grupos poblacionales (esencialmente jóvenes y adolescentes) que han crecido en un marco tecnológico digital (ordenadores, Internet, teléfonos móviles, MP3...) y cuyos usos y habilidades en relación con estos medios está completamente naturalizado. (Gertrudix, 2009)

Esta definición, resume muy bien este nuevo concepto, el cómo y el por qué estas nuevas generaciones han adoptado las redes sociales y las nuevas tecnologías como algo propio y naturalizado, ya que han pasado a formar parte de su vida "en un momento en el que la influencia de esa tecnología empieza a percibirse en nuestras generaciones más jóvenes, aquellas que han crecido y se han desarrollado en un medio plagado de 
tecnología. Esta generación está compuesta por los nativos digitales" (García, Portillo, Romo y Benito, 2007). Tal y como expresa esta cita, los vídeos son difundidos en un entorno en el que el público mayoritario empieza a ser nativos digitales, los cuales:

\section{[...] nacieron en la era digital y son usuarios permanentes de las tecnologías con} una habilidad consumada. Su característica principal es sin duda su tecnofilia. Sienten atracción por todo lo relacionado con las nuevas tecnologías [...] Estos nuevos usuarios enfocan su trabajo, el aprendizaje y los juegos de nuevas formas: absorben rápidamente la información multimedia, imágenes y videos, igual o mejor que si fuera texto. (García, Portillo, Romo y Benito, 2007)

La gran aceptación y rápido aprendizaje de los nativos digitales, como demuestra la cita de estos autores, sin duda ha sido el factor determinante para que las organizaciones, empresas o usuarios den a conocer sus productos o contenidos en el entorno online y encuentren y centren sus esfuerzos en estos tipos de públicos principalmente jóvenes con acceso ilimitado a las nuevas tecnologías en todos sus ámbitos sociales.

\subsection{BRECHA DIGITAL DE EDAD, GÉNERO Y CONDICIÓN SOCIAL}

\subsubsection{Brecha digital}

Para conocer mejor este término y como se cita en (Arenas, 2011, p. 108), "brecha digital", fue utilizada por primera vez durante la Administración Clinton (1997-2001) con la expansión de las nuevas tecnologías, haciendo referencia a la diferencia previa al acceso a las tecnologías, refiriéndonos a "conectados" y "no conectados", dicha definición es un buen ejemplo de lo anteriormente descrito dado que es la administración y los agentes sociales los primeros en hacer balance social y no comercial de impacto que pudo tener la adopción de las TIC por parte de los gobiernos y sus administraciones.

La denominada brecha digital, es uno de los temas más cercanos a la sociedad debido a que la presencia de las nuevas tecnologías ya no es un asunto de debate o de si son sostenibles o adecuadas para la población de más o menos edad, la adopción por parte de las instituciones en todos los sectores: Educativos, administrativos y sociales son 
muestra de consolidación de que las TIC han venido para quedarse y son parte fundamental en su funcionamiento. La inclusión de las TIC en los hogares y en las vidas de los ciudadanos de más edad ha sido responsabilidad de los agentes sociales y administrativos estatales encargados de que esta brecha sea lo menos notoria y perjudicial posible para las personas incluidas en ellas se familiaricen rápido con las TIC, este fenómeno ha desembocado en varias brechas identificadas como norma general en brechas de edad, de género sexual y de condición social que será analizada a lo largo del estudio.

Una de las instituciones que se han pronunciado en este aspecto, el Instituto Nacional de Estadística (principal muestra de análisis de este artículo), hace análisis y balance de este fenómeno también:

El uso de las tecnologías de información y comunicación (TIC) en los hogares ha crecido en los últimos años, si bien sigue existiendo una brecha entre los usuarios y no usuarios (brecha digital) que se puede atribuir a una serie de factores: la falta de infraestructura (en particular en las zonas rurales), la falta de conocimientos de informática y habilidades necesarias para participar en la sociedad de la información, o la falta de interés en lo que la sociedad de la información puede ofrecer. (INE, 2018)

La exhaustividad con que el INE analiza el acceso de las TIC a los hogares españoles hace que sea gran conocedor de la materia e identifique dónde se encuentran los principales factores de riesgo producidos por la brecha, como puede ser la falta de conocimiento, habilidades con las nuevas tecnologías e incluso interés en acceso o uso.

\subsubsection{Brecha digital de género}

La desigualdad efectiva entre el acceso a las nuevas tecnologías por parte de hombres y mujeres es un tema de amplio debate social, como demuestra la siguiente cita "se hace necesaria una aplicación de la igualdad real en el terreno tecnológico, que haga visible el papel de las mujeres en este campo y que socialmente las sitúe al mismo nivel que a los hombres" (Arenas, 2011, p. 100). La desigualdad se ha visto reducido por el trabajo 
de muchos profesionales y el esfuerzo de las Administraciones Públicas y casi equiparada como se verá a lo largo del análisis; la fuente o la base que se ha tomado como referencia por parte de los agentes encargados de la desaparición de dicha brecha en el ámbito público estatal sin duda ha estado basado en principios legales y jurídicos de igualdad de género, como reafirma la siguiente autora "la igualdad es el principio estructural más importante de todo Estado constitucional" (Arenas, 2011, p. 100), esta innegable premisa sin duda ha eliminado o a ayudados a eliminar este tipo de barreras ya que "un tratamiento desigual generaría un ejercicio arbitrario del poder" (Arenas, 2011, p. 100).

Un ejemplo claro de esta desigualdad efectiva que afecta al sexo femenino se encuentra en el ámbito académico, como se cita en (Gil-Juarez et al., 2001, p. 29) “nuestro entorno más inmediato no es una excepción: también en las universidades del Estado español las mujeres son claramente minoría en los estudios en el ámbito de las TIC" (Córdoba y Jofre, 2009; Pérez et. al., 2003; Fernández et. al., 2008; Sanz, 2008). Aún queda mucho camino para que esta igualdad sea equiparable entre hombres y mujeres.

No obstante, habría que realizar otro tipo de valoración o identificar, dónde se encuentran los factores que hacen que las mujeres demuestren menos interés $u$ oportunidad de acceso a las tecnologías, ya que en ocasiones los datos o índices de desigualdad de uso de las TIC entre hombres y mujeres, no está determinada al acceso o al derecho de uso como se ha tratado anteriormente, si no al interés que hay que despertar entre las féminas del uso y acceso profesional o académico de las nuevas tecnologías.

\subsubsection{Brecha digital de edad}

El caso de la brecha digital de edad es un asunto de primer orden por muchos factores, uno de ellos es el progresivo aumento de la población de más edad en nuestro país, ya que "la población de mayor edad en España, [...] experimentará de forma general un crecimiento más rápido que la media del resto de países europeos" (Peral, Villarejo y Arenas, 2017, p. 67), este factor imparable y continuo dado que los ciclos naturales de vida son siempre a tender a envejecer, habida cuenta, en un entorno digital y 
tecnológico, será siempre una asignatura pendiente o un reto para las personas ya que la adaptación y el aprendizaje de los nuevos medios emergentes en continua. La adopción por parte de los gobiernos, sus administraciones y el entorno social, está condicionado a las nuevas tecnologías como se ve a lo largo del presente estudio y en numerosos artículos de esta temática, por tanto "las TIC son un importante motor de modernización económica y social, y su empleo puede suponer, para la población de mayor edad, un impacto significativo en su calidad de vida" (Peral, Villarejo y Arenas, 2017, p. 67).

Los hábitos de consumo de la población de más edad están determinados al factor del envejecimiento, esta causa es fundamental para entender los procesos que se producen como el aprendizaje, la adaptación o la aceptación a los nuevos medios ya que "a medida que envejecemos, comenzamos a percibir un deterioro de las condiciones físicas" (Peral, Villarejo y Arenas, 2017, p. 72), esta variable "pueden diferenciar el comportamiento de los mayores ante el uso de servicios en línea elegidos" (Peral, Villarejo y Arenas, 2017, p. 71). Sin embargo, esa madurez y experiencia de vida les hace contar con otra visión de los medios "los mayores [...] no usan Internet cuando la información que necesitan está disponible a través de medios tradicionales" (Peral, Villarejo y Arenas, 2017, p. 71) y en cierta medida un uso más sostenible y responsable de las TIC e incluso "disfrutan con experiencia nuevas y no les produce ansiedad interactuar con la tecnología" (Peral, Villarejo y Arenas, 2017, p. 76), y consiguiendo con su uso llevar las TIC al ámbito familiar "las aplicaciones de comunicación permiten también un nivel de comunicación interfamiliar que obra en beneficio de la tranquilidad de todos los afectados, al mismo tiempo que evita la exclusión del colectivo" (Caldevilla y García, 2015, p. 31). De hecho, el incremento de la población de más edad en el uso de internet del 47,7\% en 2012 al 74,5 \% en 2017 de la población mayor de entre 55 a 64 (INE, 2017), este factor es un indicador de que los mayores han sabido y saben adaptarse a estos nuevos medios e incluirlos en su vida cotidiana, a través del uso de web's, App's, ordenadores y las distintas formas de conexión.

\subsubsection{Brecha digital de condición social}


El entorno digital se ha desarrollado en un mundo paralelo al progreso y acceso a las nuevas tecnologías, pero a niveles de riqueza ha provocado lo que se conoce como la brecha digital de condición social que "puede ser definida en términos de la desigualdad de posibilidades que existen para acceder a la información, al conocimiento y la educación mediante las nuevas tecnologías" (Almenara, 2004), esta definición dada por Almenara resume muy bien la naturaleza de este fenómeno, provocando que "aquellos ciudadanos que no estén cualificados para el uso de las TIC tendrán una mayor probabilidad de ser marginados" (Arenas, 2011, p. 107), estas diferencias sociales, ya no solo pueden afectar al aprendizaje $u$ acceso a la información, si no que una desactualización en el desarrollo de las nuevas tecnologías por parte de los ciudadanos con menos recursos se traslada también al mundo laboral ya que "las TIC afectan de forma muy profunda al trabajo, las relaciones laborales y la estructura social" (Olarte, 2017, p. 286) provocando peores competencias laborales a estos ciudadanos en comparación con el resto que sí pueden acceder a ellas, contradiciendo en cierta medida los avances y progresos para los que están creadas las TIC ya que "las TIC han generado expectativas de mejora social, al posibilitar, en términos teóricos, el acceso universal a la información, fortalecer la sociedad civil y la democracia, avanzar en la inclusión social, optimizar recursos, brindar nuevas oportunidades culturales, generar nuevos empleos y más progreso social" (Olarte, 2017, p. 287)

En un entorno "en el que la accesibilidad universal, el acceso a internet y la extensión de la infraestructura de la información y la comunicación juegan un papel determinante en este sentido" (Olarte, 2017, p. 287), se ha producido una bifurcación en la problemática que se ha generado con la brecha digital de condición social; por un lado, se entiende esta brecha como el no acceso a las nuevas tecnologías, con las repercusiones en materia de desigualdad tiene en la sociedad como se está viendo a lo largo del estudio, sin embargo se está obviando en cierta medida la desigualdad o la brecha que se produce fruto del mercado internacional de productos relacionados con las TIC, siendo "un elemento básico para el mantenimiento del modelo económico neoliberal basado en el libre mercado" (Arriazu, 2015, p. 226), este factor hace que la diferencia esté marcada por el comportamiento del comercio y no de la falta de acceso 
debido al alto precio que los productos pueden alcanzar en el mercado, siendo el desarrollo de las TIC y los productos que contiene los causantes de la desigualdad "el consumo de bienes y servicios tecnológicos como un elemento de representación, estructuración y distinción social" (Arriazu, 2015, p. 227), siendo por tato esta contingencia un efecto del mercado.

\section{MÉTODO}

\subsection{MÉTODO}

Para el análisis de las muestras se empleará la metodología cuantitativa, "el enfoque cuantitativo utiliza la recolección y el análisis de datos para contestar preguntas de investigación (...) y confía en la medición numérica, el conteo y frecuentemente en el uso de la estadística para establecer con exactitud patrones de comportamiento en una población" (Sampieri, Collado y Baptista, 2006, p. 9).

Será objeto de análisis los resultados sobre los datos de las encuestas del Instituto Nacional de Estadística de España, este análisis, unido a lo descrito en el marco teórico, ayudarán a comprender y entender mejor el objeto del estudio que se aborda.

\subsection{MUESTRA}

La primera, es una muestra de 6 tablas de elaboración propia de un total de 69 encuestas sobre los resultados nacionales del Instituto Nacional de Estadística realizadas a la población española sobre equipamiento y uso de Tecnologías de la Comunicación y la Información en los hogares durante el 2017, se ha elegido esta relación de encuestas ya que se ha considerado que la información que contienen es la más idónea y representativa de los usos que la ciudadanía hacen de la nuevas tecnologías de manera cotidiana y cercana a la realidad social y de los hogares, todo ello con el fin de entender y comprender mejor los conceptos de nativo digital y brecha digital y los usos que las distintas generaciones hacen de los medios digitales e Internet, por tanto se ha segmentado la información según las siguientes variables divididas de la siguiente forma: 
a) Franja o brecha de género (hombre y mujer), en este segmento se muestra los datos en porcentaje (\%) y número de personas de hombres y mujeres que hacen uso de las nuevas tecnologías e Internet.

b) Franja o brecha de edad, en esta muestra segmentada por edad, se encuentran incluidas las personas insertas en la generación que se denomina nativos digitales y los incluidos en la brecha digital, los intervalos de edad quedan comprendidos la siguiente forma:

- Entre 16 y 24 años.

- $\quad$ Entre 55 y 64 años.

c) Franja o brecha de condición social, en dicha muestra se recogen los datos de los distintos usos y número de personas que hacen uso de los medios digitales e Internet atendiendo a la siguiente relación de volumen de ingresos familiares:

- Ingresos mensuales netos del hogar al que pertenecen: Menos de 900 euros.

- Ingresos mensuales netos del hogar al que pertenecen: Más de 2.500 euros.

Los análisis de las encuestas están basados en la diversidad de información de carácter muy riguroso sobre los hábitos que la población española tiene sobre haber utilizado alguna vez el ordenador y personas que han utilizado alguna vez Internet y las personas que reciben o envían correo electrónico y participar en redes sociales (creando un perfil de usuario, enviando mensajes u otras contribuciones a Facebook, Twitter, Tuenti, etc.).

\section{RESULTADOS}

Los resultados cuantitativos serán analizados de la siguiente forma.

Los datos están marcados en negrita, dentro de la misma celda subdividida con una línea ascendente y con un leve sombreado gris perla para dotar a los resultados de valores diferenciadores para mejor lectura, diferenciación y comprensión de los datos que darán consistencia y argumentación al estudio llevado a cabo, las celdas contienen el porcentaje y la representación numérica calculadas con la siguiente fórmula: 
№ de viviendas, personas o equipamiento $=$ Total de viviendas, personas 0 equipamiento $\times 100 /(\%)$

De forma específica se analizará cada franja o brecha de edad, género y condición social, destacando o resumiendo las observaciones más importantes con un breve texto al pie de cada cuadro, los criterios que se han seguido para cada franja o brecha han sido los siguientes:

Para el análisis de las franjas de edad, se muestran:

Las personas incluidas en la brecha digital, para este segmento se va a considerar el intervalo de edad comprendida entre 55 a 64, se ha considerado esta muestra, ya que son personas de edad adulta o no nacidas en el entorno digital, pero que se encuentran en edad laboral (artículos 6 y 7 del Estatuto de los Trabajadores) y con plena capacidad de obrar (artículo 322 de Código Civil) y que en cierto modo han tenido saber adaptarse o adquirir conocimientos adaptados al nuevo entorno digital en sus ámbitos laborales, personales, etc.

Por otro lado, se considerará a las personas nacidas en el entorno digital o nativos digitales a la franja de edad comprendida entre 16 y 24 años, ya que sí son personas nacidas o considerando que, aunque no sean nacidas, sí han crecido cerca de las nuevas tecnologías, el entorno digital e Internet y en todos los ámbitos sociales en los que desarrolla su vida como las actividades laborales (artículos 6 y 7 del Estatuto de los Trabajadores), de amistad, educativas y pero que no cuentan con todas las capacidades de obrar como se regula en el Código Civil por la limitación interpuesta debido a la edad.

Para el análisis de la franja de género o las diferencias que existan entre hombres y mujeres serán analizadas de forma porcentual y numérica, estableciendo las diferencia que puedan existir entre ambos géneros. 
Para el análisis de la franja social, se tendrá en cuenta el volumen de ingresos netos mensuales en los hogares, para ello se tendrá en cuenta las diferencias entre los hogares con menos ingresos, es decir, ingresos mensuales netos del hogar al que pertenecen: Menos de 900 euros y los hogares de mayor ingresos, por tanto Ingresos mensuales netos del hogar al que pertenecen: Más de 2.500 euros, con el fin de tener datos en porcentaje u numéricos que muestren la verdadera diferencia o comparativa que pueda diferencia que pueda existir entre ambas franjas.

Los cuadros de análisis sobre los resultados estadísticos en porcentaje y numéricos quedan estructurados de la siguiente forma:

Cuadro 1. Resultados sobre el uso de productos TIC por característica demográficas, género y tipo de producto.

\begin{tabular}{|c|c|c|c|}
\hline velose & Total de personas & $\begin{array}{c}\text { Personas que han } \\
\text { utilizado alguna vez el } \\
\text { ordenador }\end{array}$ & $\begin{array}{c}\text { Personas que han } \\
\text { utilizado alguna vez } \\
\text { Internet }\end{array}$ \\
\hline Total de personas & 34.449 .634 & 81,9 & 86,0 \\
\hline \multirow[t]{2}{*}{ Sexo: Hombre } & 17.105 .097 & 84,0 & 86,8 \\
\hline & & 14.368.281 & 14.847 .22 \\
\hline \multirow[t]{2}{*}{ Sexo: Mujer } & 17.344 .537 & 79,8 & 85,2 \\
\hline & & 13.8 & \\
\hline \multirow[t]{2}{*}{ De 16 a 24} & 4.009 .028 & 97,5 & 98,0 \\
\hline & & & \\
\hline \multirow[t]{2}{*}{ De 55 a 64} & 5.791 .229 & 69,4 & 76,1 \\
\hline & & & \\
\hline
\end{tabular}

Fuente: Elaboración propia con datos del Instituto Nacional de Estadística (INE). 
Se puede apreciar que existe igualdad entre hombres y mujeres en el uso de Internet y el ordenador. De igual modo se puede observar que las personas con edad comprendida entre los 16 y 24 años hacen mayor uso del ordenador e Internet que las personas de entre 55 a 64 años.

Cuadro 2. Resultados sobre el uso de productos TIC y tipo de producto en las viviendas atendiendo al volumen de ingresos mensuales netos del hogar.

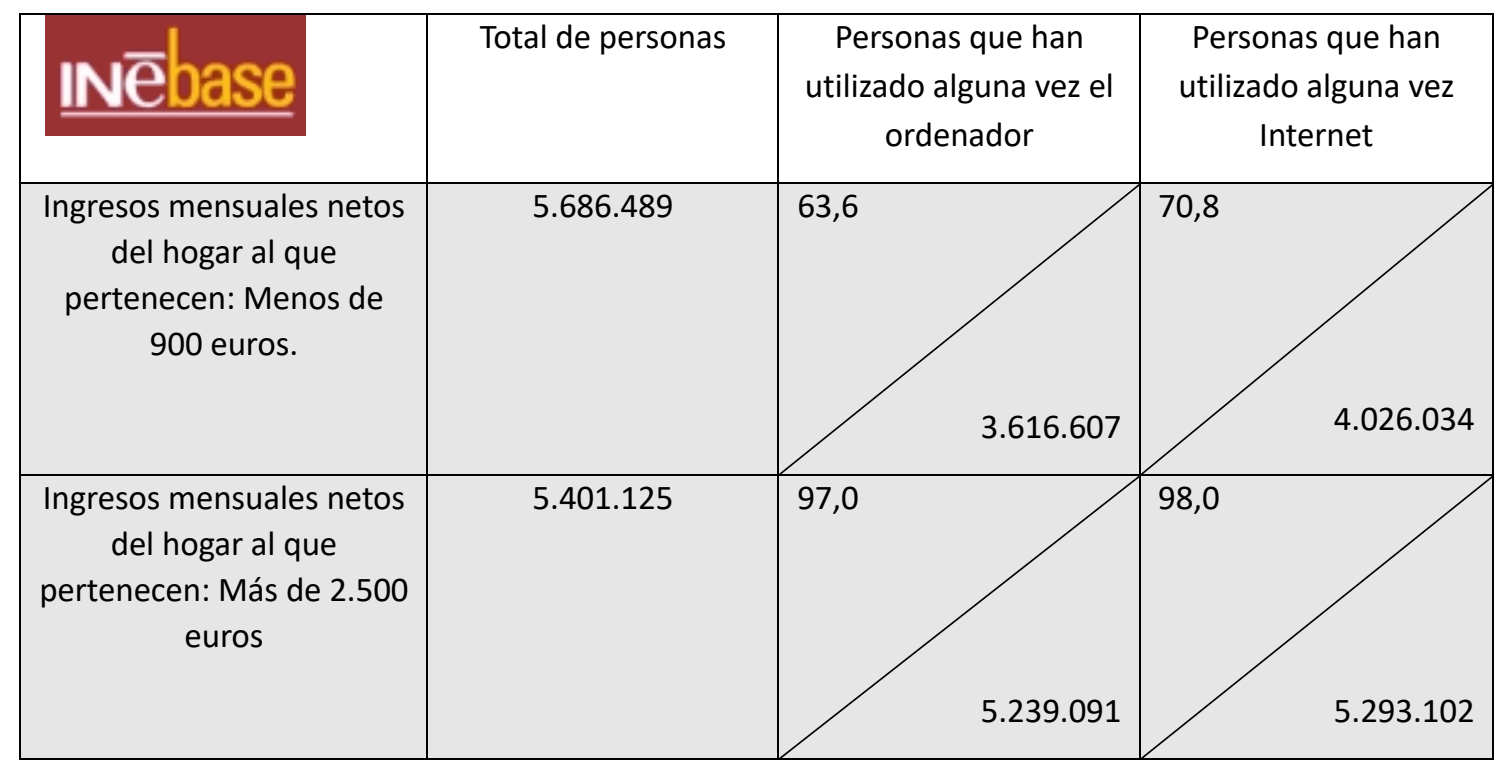

Fuente: Elaboración propia con datos del Instituto Nacional de Estadística (INE).

Se puede ver que las personas con un mayor ingreso familiar hacen más uso de los ordenadores e Internet que las familias con menos ingreso.

Cuadro 3. Resultados sobre los servicios de Internet usados por motivos particulares en los últimos 3 meses y naturaleza del servicio de comunicación y acceso a la información por características demográficas y de género.

\begin{tabular}{|l|c|c|c|}
\hline INege & $\begin{array}{c}\text { Total de personas } \\
\text { que han utilizado } \\
\text { Internet en los } \\
\text { últimos 3 meses }\end{array}$ & $\begin{array}{c}\text { Recibir o enviar } \\
\text { correo electrónico }\end{array}$ & $\begin{array}{c}\text { Participar en redes sociales } \\
\text { (creando un perfil de } \\
\text { usuario, enviando mensajes } \\
\text { u otras contribuciones a } \\
\text { Facebook, Twitter, Tuenti, }\end{array}$ \\
\hline
\end{tabular}

RAEIC, Revista de la Asociación Española de Investigación de la Comunicación vol. 6, núm. 11 (2019), 203-223 


\begin{tabular}{|c|c|c|c|}
\hline & & & etc.) \\
\hline Total de personas & 29.145 .163 & & \\
\hline Sexo: Hombre & 14.619 .401 & 79,9 \\
\hline Sexo: Mujer & 14.525 .763 & \\
\hline De 16 a 24 & 3.928 .313 \\
\hline
\end{tabular}

Fuente: Elaboración propia con datos del Instituto Nacional de Estadística (INE).

Las mujeres participan más en las redes sociales que los hombres, de la misma forma se constata que los nativos digitales usan más los servicios ofrecidos en Internet.

Cuadro 4. Resultados sobre los servicios de Internet y naturaleza del servicio de comunicación y acceso a la información atendiendo al volumen de ingresos mensuales netos del hogar.

\begin{tabular}{|c|c|c|c|}
\hline uese & $\begin{array}{c}\text { Total de personas } \\
\text { que han utilizado } \\
\text { Internet en los } \\
\text { últimos 3 meses }\end{array}$ & $\begin{array}{c}\text { Recibir o enviar correo } \\
\text { electrónico }\end{array}$ & $\begin{array}{c}\text { Participar en redes } \\
\text { sociales (creando un } \\
\text { perfil de usuario, } \\
\text { enviando mensajes u } \\
\text { otras contribuciones a } \\
\text { Facebook, Twitter, } \\
\text { Tuenti, etc.) }\end{array}$ \\
\hline $\begin{array}{c}\text { Ingresos mensuales } \\
\text { netos del hogar al que } \\
\text { pertenecen: Menos de } \\
900 \text { euros. }\end{array}$ & 3.888 .669 & 65,9 & 68,5 \\
\hline
\end{tabular}

RAEIC, Revista de la Asociación Española de Investigación de la Comunicación vol. 6, núm. 11 (2019), 203-223 


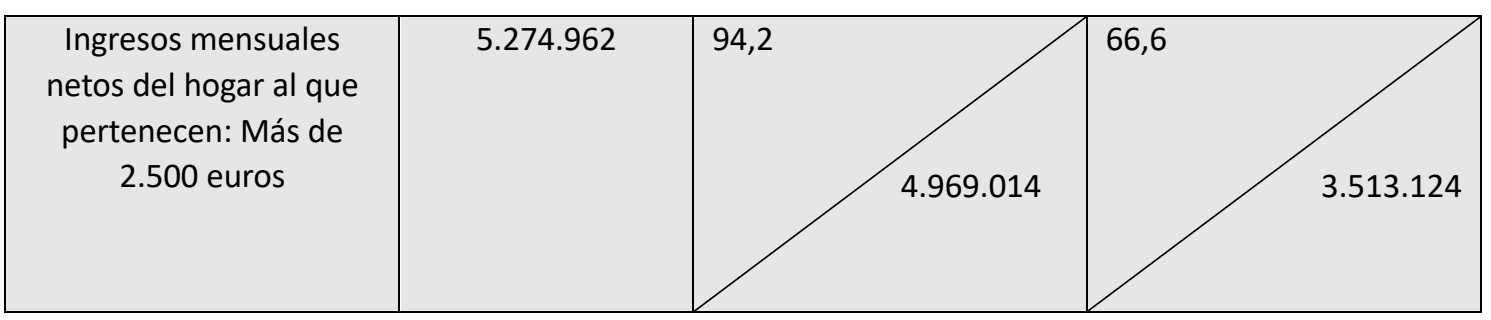

Fuente: Elaboración propia con datos del Instituto Nacional de Estadística (INE).

Las familias con mayor ingreso usan más los servicios de Internet, salvo en el uso de redes sociales que es mayor el porcentaje perteneciente a las familias con menores ingresos.

\section{DISCUSIONES Y CONCLUSIÓN}

Haciendo una comparativa entre los datos pertenecientes a las encuestas del Instituto Nacional de Estadística sobre equipamiento y uso de Tecnologías de la Comunicación y la Información en los hogares durante el 2017 y del año 2012, se extraen las siguientes conclusiones:

En el caso de la brecha de género, puede observarse que le número de usuarios de ordenador y de Internet ha aumentado entre los años 2012 y 2017, sin embargo, en número de mujeres que usan el ordenador se ha visto incrementado en detrimento de los hombres en ambos años, concluyendo que las mujeres demuestran menos grado de confianza que los hombres al usar Internet.

\begin{tabular}{|c|c|c|}
\hline 2012 & Uso del ordenador & Uso de Internet \\
\hline Hombres & $79,8 \%$ & $75,5 \%$ \\
\hline Mujeres & $75,2 \%$ & $70,8 \%$ \\
\hline \multicolumn{3}{|l|}{2017} \\
\hline Hombres & $84 \%$ & $86,8 \%$ \\
\hline Mujeres & $85,2 \%$ & $79,8 \%$ \\
\hline
\end{tabular}

Fuente: Elaboración propia con datos del Instituto Nacional de Estadística (INE).

El número de personas insertas en la brecha de edad se ha visto incrementado y casi doblada en el uso de Internet desde 2012 a 2017, demuestra que las personas de más 
edad están sabiendo adaptarse a las TIC, el número usuarios nativos digitales apenas ha variado.

\begin{tabular}{|c|c|c|}
\hline 2012 & Uso del ordenador & Uso de Internet \\
\hline $16-24$ años & $98,9 \%$ & $47 \%$ \\
\hline $55-64$ años & $53,1 \%$ & $98 \%$ \\
\hline $16-24$ años & & $76,1 \%$ \\
\hline $55-64$ años & $97,5 \%$ & \\
\hline
\end{tabular}

Fuente: Elaboración propia con datos del Instituto Nacional de Estadística (INE).

En el caso de la brecha de condición social puede observarse que las familias con menores ingresos no han cambiado en los hábitos de uso del ordenador, sí se ha visto reducido en número de internautas de esta condición social, cabe destacar que las familias de mayor ingreso no han cambiado hábito desde 2012 a 2017, provocado por las nuevas formas de conexión y acceso, que han podido encarecer los productos. La variable ingresos fue analizada en intervalos distintos a los del 2017 considerando en el año 2012 el ingreso mínimo familiar en 1100 euros y el ingreso máximo familiar en más de 2700 euros, no obstante se ha considerado para el estudio igual de representativo para el análisis.

\begin{tabular}{|l|c|c|}
\hline 2012 & Uso del ordenador & Uso de Internet \\
\hline Ingresos menos de 1100 euros & $63,6 \%$ & $98,8 \%$ \\
\hline Ingresos más de 2700 euros & $97 \%$ & $98 \%$ \\
\hline 2017 & $63,6 \%$ & $70,8 \%$ \\
\hline Ingresos menos de 900 euros & $97 \%$ & $98 \%$ \\
\hline Ingresos más de 2500 euros & & \\
\hline
\end{tabular}

Fuente: Elaboración propia con datos del Instituto Nacional de Estadística (INE).

RAEIC, Revista de la Asociación Española de Investigación de la Comunicación vol. 6, núm. 11 (2019), 203-223 
La siguiente de la comparativa es de los datos sobre los servicios de Internet usados por motivos particulares en los últimos 3 meses y naturaleza del servicio de comunicación y acceso a la información por características demográficas y socioeconómicas, de las encuestas del INE entre los años 2012 y 2017, sobre el uso de las redes sociales y e-mails, considerando que son herramientas de uso muy cotidianas y representativas, sacando las siguientes conclusiones:

En el caso de la brecha de género puede verse que las mujeres han pasado a usar más las redes sociales que los hombres desde el año 2012 a 2017, cabe señalar que el uso del correo electrónico se ha visto reducido en ambos sexos en detrimento de las redes sociales, en un efecto de aceptación de los social media por parte de la ciudadanía.

\begin{tabular}{|c|c|c|}
\hline 2012 & Uso de redes sociales & Uso de mail \\
\hline Hombres & $62,1 \%$ & $89,1 \%$ \\
\hline Mujeres & $60,2 \%$ & $87,4 \%$ \\
\hline \multicolumn{3}{|l|}{2017} \\
\hline Hombres & $65,2 \%$ & $81,9 \%$ \\
\hline Mujeres & $70,6 \%$ & $77,8 \%$ \\
\hline
\end{tabular}

Fuente: Elaboración propia con datos del Instituto Nacional de Estadística (INE).

En el caso de la brecha de edad y nativos digitales, en este caso particular, posiblemente sea el más reseñable de todos debido a que el número de nativos digitales que usan redes sociales y usan el e-mail se ha visto disminuido desde el año 2012 a 2017, en el caso de las personas insertas en la brecha digital se ha comportado de la misma forma en el caso de las redes sociales pero ha duplicado el número de usuarios de e-mail en detrimento de las redes sociales, debido a que han considerado más eficiente la forma de comunicación vía mail, puesto que los datos son casi directamente proporcionales entre las RRSS y el correo electrónico, en un efecto de normalización tras el boom de los social media.

\begin{tabular}{|c|c|c|}
\hline 2012 & Uso de redes sociales & Uso de mail \\
\hline $16-24$ años & $94,7 \%$ & $90,2 \%$ \\
\hline
\end{tabular}




\begin{tabular}{|c|c|c|}
\hline \multicolumn{1}{|c|}{$55-64$ años } & $83,4 \%$ & $34,1 \%$ \\
\hline 2017 & & $84,7 \%$ \\
\hline $16-24$ años & $90 \%$ & $67 \%$ \\
\hline $55-64$ años & $46,2 \%$ & \\
\hline
\end{tabular}

Fuente: Elaboración propia con datos del Instituto Nacional de Estadística (INE).

En el caso de la brecha de condición social ocurre algo similar a la tabla anteriormente comentada, ambos casos familiares han disminuido en el uso de las redes sociales, destacable el caso de las familias de más renta demostrando más confianza en el uso del e-mail y disparándose el incremento de uso de esta en las familias de mayor ingreso. (Variable ingreso de 2012 ídem a la anterior).

\begin{tabular}{|l|c|c|}
\hline 2012 & Uso de redes sociales & Uso de mail \\
\hline Ingresos menos de 1100 euros & $80,6 \%$ & $58,9 \%$ \\
\hline Ingresos más de 2700 euros & $96,4 \%$ & $61 \%$ \\
\hline 2017 & $68,5 \%$ & $65,9 \%$ \\
\hline Ingresos menos de 900 euros & $65,9 \%$ & $94,2 \%$ \\
\hline Ingresos más de 2500 euros & & \\
\hline
\end{tabular}

Fuente: Elaboración propia con datos del Instituto Nacional de Estadística (INE).

De otro lado se ha podido observar el bajo acceso a los recursos tecnológicos y productos de las familias de menor ingresos debido al bajo volumen de ingresos y el costo de los productos que en la mayoría de los casos las familias no pueden soportar, provocado por los altos precios del mercado y la continua actualización y creación de nuevos productos que se producen en el comercio electrónico, como se puede observar en el cuadro 2 sobre los el tipo de equipamiento digital relacionados con los ingresos mensuales netos del hogar.

Como conclusión general sobre el uso de las TIC por parte de la población española durante el 2017, se puede decir que el incremento en todas las variables analizadas, sexo, edad y condición social demuestra que de forma paulatina las TIC están penetrando en los hogares y en las vidas en un efecto de adaptación y aceptación, no obstante no se puede dejar pasar por alto que este incremento es menor en 
comparación con las familias de menor ingresos que las familias con mayor ingreso se demuestra así que el mercado tecnológico en un efecto de continua actualización provoca que cierto material quede inservible y alcance precios prohibitivos en algunos caso para las familias con menor poder adquisitivo, siendo esta la variable que había que mejorar. Se ha descartado otras variables de intervalo de edad ya que no han considerado representativas para el presente estudio.

\section{REFERENCIAS BIBLIOGRÁFICAS}

Almenara, J. C. (2004). Reflexiones sobre la brecha digital y la educación. En F. J. Soto y J. Rodríguez (coords.), Tecnología, educación y diversidad: retos y realidades de la inclusión social. Murcia: Consejería de Educación y Cultura.

Arenas, M. (2011). Brecha digital de género: La mujer y las nuevas tecnologías. En Anuario de la Facultad de Derecho. Alcalá: Universidad de Alcalá.

Arriazu, R. (2015). La incidencia de la brecha digital y la exclusión social tecnológica: el impacto de las competencias digitales en los colectivos vulnerables. Praxis sociológica, (19), 225-240

Caldevilla, D. y García, E. (2015). APP para la tercera edad utilidad clases y valor social. Revista de Ciencias de la Información, (36), 26-36.

Enjoy Life (05 de julio de 2017). Este es el video más visto de la historia de Facebook \#video \#viral \#facebook. Enjoy Life. Recuperado de http://enjoylife.com.ar/novedades/este-video-mas-visto-la-historia-facebook/

Excelsior (29 de marzo de 2018). El video más visto en la historia de Facebook. Excelsior. Recuperado de https://www.excelsior.com.mx/trending/el-video-mas-vistoen-la-historia-de-facebook/1229417

Galeano, S. (31 de enero de 2019). El número de usuarios de Internet en el mundo crece un 9,1\% y alcanza los 4.388 millones (2019). Marketing 4 Ecommerce.

Recuperado de https://marketing4ecommerce.net/usuarios-internet-mundo/

García, F., Portillo, J., Romo, J. y Benito, M. (2007). Nativos digitales y modelos de aprendizaje. Trabajo presentado en el IV Simposio Pluridisciplinar sobre Diseño, Evaluación y Desarrollo de Contenidos Educativos Reutilizables, Bilbao. Recuperado de http://ceur-ws.org/Vol-318/Garcia.pdf

Gertrudix-Barrio, M. (2009). Nativos digitales. Presentación. Icono 14, 7(1), 1-6.

Gil, A., Vitores, A., Feliu, J. y Vall-Lovera, M. (2011). Brecha digital de género: Una revisión y una propuesta. Education in the knowledge society, 12(2), 25-53. 
Insituto Nacional de Estadística (2017). Equipamiento y uso de Tecnologías de la Comunicación y la Información en los hogares durante el 2017. Recuperado de https://www.ine.es/prensa/tich_2017.pdf

Olarte, S. (2017). Brecha digital, pobreza y exclusión social. Temas laborales: Revista andaluza de trabajo y bienestar social, (138), 285-313.

Peral, B., Arenas, J. y Villarejo-Ramos, A. F. (2015). De la brecha digital a la brecha psico-digital: Mayores y redes sociales. Comunicar: Revista científica iberoamericana de comunicación y educación, XXIII(45), 57-64.

Real Decreto Legislativo 2/2015, de 23 de octubre, por el que se aprueba el texto refundido de la Ley del Estatuto de los Trabajadores. Boletín Oficial del Estado. Madrid, 24 de octubre de 2015, núm. 255.

Real Decreto de 24 de julio de 1889 por el que se publica el Código Civil. Boletín Oficial del Estado. Madrid, 25 de julio de 1889, núm. 206.

Sampieri, R., Collado, C. y Baptista, P. (2006). Metodología de la investigación. México: McGraw-Hill. 